\section{Material, infektiöses}

W. G. Guder

München, Deutschland

Synonym(e) Ansteckungsgefährliche Stoffe; Untersuchungsgut, gefährliches, infektiöses

Englischer Begriff infectious substance; biohazard; infectious hazard

Definition Als infektiöses Material werden alle Stoffe gekennzeichnet, von denen bekannt oder anzunehmen ist, dass sie Krankheitserreger enthalten, die bei Menschen oder Tieren infektiöse Krankheiten verursachen und die in der Lage sind, an der Stelle ihres Freiwerdens Krankheiten auf zufällig anwesende Personen oder Tiere zu übertragen. Krankheitserreger können Mikroorganismen (Bakterien, Viren, Pilze etc.), aber auch endogene Moleküle wie Prionen sein, welche die spongiforme Enzephalopathie übertragen.

Beschreibung Infektiöses Material im Sinne der UN-Definition stellen alle diagnostisch und zum Zwecke der Erforschung von Patienten (und Tieren) entnommenen Materialien sowie ihre Ausscheidungen mit potenziell infektiösen Erregern dar. Sie müssen während des Umgangs, des Transports und der Entsorgung entsprechend gekennzeichnet und behan- delt werde. Die Regeln zum Umgang und zum Versand sind in internationalen Gesetzen und Richtlinien geregelt. Lokale Verhaltensmaßnahmen am Arbeitsplatz werden durch Gefahrgutbeauftragte vorgeschrieben und überwacht.

In der $>$ Laboratoriumsmedizin hat dies nicht nur in der mikrobiologischen Diagnostik, sondern bei allen mit menschlichem und tierischem Untersuchungsmaterial umgehenden Personen weitgehende Konsequenzen, da in Unkenntnis des Patienten zur Sicherheit jede Probe wie infektiöses Material zu behandeln ist. Dies gilt insbesondere im Umgang mit Kulturen von Krankheitserregern, infektiösen Ausscheidungen und flüchtigen Stoffen mit infektiösem Material (z. B. Sputum von Tuberkuloseerkrankten).

Die Vorschriften für den Transport dieser Stoffe sind in nationalen Gesetzen geregelt (s. a. - Versand von Proben).

\section{Literatur}

Deutsche Post AG (2010) Regelungen über den Postversand von medizinischem und biologischem Untersuchungsgut. http:/www.suesse.de/ service/postversand-medizinisches-biologisches-untersuchungsgut/

Guder WG, Narayanan S. (2015) Sample transport, treatment after arrival, storage and disposal. In Guder WG, Narayanan S. Pre-Examination Procedures in Laboratory Diagnostics. Berlin,New York: Walter de Gruyter, S. 251-263

WHO (2007-2008) Guidance on regulation for the transport of infectious substances. World Health Organization (WHO), Geneva 